\title{
Cellular Scaling Rules for the Brains of Marsupials: Not as "Primitive" as Expected
}

\author{
Sandra E. Dos Santos ${ }^{a}$ g, h Jairo Porfirio ${ }^{a} \quad$ Felipe B. da Cunha ${ }^{a}$ Paul R. Manger \\ William Tavares $^{b}$ Leila Pessoa ${ }^{b}$ Mary Ann Raghantid ${ }^{d}$ Chet C. Sherwood ${ }^{e, f}$ \\ Suzana Herculano-Houzel ${ }^{\mathrm{a}, \mathrm{g}} \mathrm{h}$ \\ ${ }^{\mathrm{a}}$ Instituto de Ciências Biomédicas and ${ }^{\mathrm{b}}$ Instituto de Biologia, Universidade Federal do Rio de Janeiro, \\ Rio de Janeiro, Brazil; ' $S$ Chool of Anatomical Sciences, University of the Witwatersrand, Johannesburg, South Africa; \\ ${ }^{\mathrm{d}}$ Department of Anthropology, Kent State University, Kent, $\mathrm{OH},{ }^{e}$ Department of Anthropology and ${ }^{\mathrm{f}}$ Center for the \\ Advanced Study of Human Paleobiology, The George Washington University, Washington, DC, and Departments of \\ gPsychology and hiological Sciences, Vanderbilt University, Nashville, TN, USA
}

\section{Keywords}

Evolution - Allometry · Marsupials · Brain size · Numbers of neurons $\cdot$ Glia/neuron ratio $\cdot$ Cortical expansion

\footnotetext{
Abstract

In the effort to understand the evolution of mammalian brains, we have found that common relationships between brain structure mass and numbers of nonneuronal (glial and vascular) cells apply across eutherian mammals, but brain structure mass scales differently with numbers of neurons across structures and across primate and nonprimate clades. This suggests that the ancestral scaling rules for mammalian brains are those shared by extant nonprimate eutherians but do these scaling relationships apply to marsupials, a sister group to eutherians that diverged early in mammalian evolution? Here we examine the cellular composition of the brains of 10 species of marsupials. We show that brain structure mass scales with numbers of nonneuronal cells, and
}

numbers of cerebellar neurons scale with numbers of cerebral cortical neurons, comparable to what we have found in eutherians. These shared scaling relationships are therefore indicative of mechanisms that have been conserved since the first therians. In contrast, while marsupials share with nonprimate eutherians the scaling of cerebral cortex mass with number of neurons, their cerebella have more neurons than nonprimate eutherian cerebella of a similar mass, and their rest of brain has fewer neurons than eutherian structures of a similar mass. Moreover, Australasian marsupials exhibit ratios of neurons in the cerebral cortex and cerebellum over the rest of the brain, comparable to artiodactyls and primates. Our results suggest that Australasian marsupials have diverged from the ancestral Theria neuronal scaling rules, and support the suggestion that the scaling of average neuronal cell size with increasing numbers of neurons varies in evolution independently of the allocation of neurons across structures.

\section{KARGER}

(c) 2017 S. Karger AG, Basel

E-Mail karger@karger.com

www.karger.com/bbe
Suzana Herculano-Houzel or Sandra E. Dos Santos

Department of Psychology, Vanderbilt University

11121 st Avenue South

Nashville, TN 37240-7817 (USA)

E-Mail suzana.herculano@vanderbilt.edu or sandra.e.dos.santos@vanderbilt.edu 


\section{Introduction}

What are the rules that govern how numbers of cells and cell size vary within brain regions in the evolution and diversification of mammalian lineages? Early comparative studies of the cellular composition of the brain implicitly considered that all mammalian brains were built the same way, with a shared relationship between the volume or mass of the cerebral cortex $\left(\mathrm{M}_{\mathrm{CX}}\right)$ and its density of neurons and glia/neuron ratio [Haug, 1987; Stolzenburg et al., 1989]. The proportionality between brain structure mass and number of nonneuronal (glial and vascular) cells is indeed shared not only across the more than 40 eutherian species examined so far but also across the brain structures analyzed, indicating that the relationship between numbers of nonneuronal cells and the mass of the structures they form has been maintained for at least 110 million years of evolution [HerculanoHouzel, 2014; Herculano-Houzel et al., 2014a].

In contrast, new data on the numbers of neurons that compose different brain structures in primates, glires, eulipotyphlans, afrotherians, and artiodactyls [HerculanoHouzel et al., 2006, 2007, 2011, 2014b, Sarko et al., 2009; Gabi et al., 2010; Kazu et al., 2014; Neves et al., 2014; reviewed in Herculano-Houzel et al., 2015a] have shown that different scaling relationships apply between brain structure size and number of neurons both across structures and across mammalian orders. Still, while there is variation in phylogeny, some neuronal scaling rules are indeed shared by a number of mammalian orders, suggesting that they might represent ancestral scaling rules for mammalian brains [Herculano-Houzel et al., 2014a]. For example, the mass of the cerebral cortex as well as its neuronal density scale as functions of the number of cortical neurons that are shared across Afrotheria, Glires, Eulipotyphla, and Artiodactyla - but not primates. The latter are characterized by an evolutionarily derived scaling relationship that results in more cortical neurons building a given cortical volume compared to nonprimates [Herculano-Houzel et al., 2014a, 2015a]. A similar pattern is found across primate and nonprimate "rest of brain" (RoB; the ensemble of brainstem, diencephalon, and striatum), with more neurons fitting in the primate RoB than in nonprimate structures of a similar mass. Likewise, the mass of the cerebellum as well as its density of neurons scale as shared functions of the number of cerebellar neurons in Afrotheria, Glires, and Artiodactyla, but not in Eulipotyphla and Primata, which appear to have diverged (without the elephant [Herculano-Houzel et al., 2014a, 2015a]).

Marsupial Brains Are Not Primitive
Interestingly, regardless of the different scaling of cerebral cortical and cerebellar mass across primates, eulipotyphlans, and other eutherians as these structures gain neurons, neurons are added in evolution at an apparently constant rate of 4 neurons in the cerebellum $(\mathrm{Cb})$ to every neuron in the cerebral cortex $(\mathrm{Cx})$ in a manner that applies across all eutherian species analyzed so far (with the exception of the elephant [Herculano-Houzel, 2010; Herculano-Houzel et al., 2014a]). In contrast, while glires, eulipotyphlans, and small afrotherians share an average ratio of 2 neurons in the $\mathrm{Cx}$ for every neuron in the RoB, primates and artiodactyls have increased ratios of neurons in the Cx over the RoB [Herculano-Houzel et al., 2014a].

Based on the principle of parsimony, we have proposed that those allometric scaling relationships, or scaling rules, shared by the majority of extant eutherian mammalian species analyzed must also have applied to ancestral mammalian brains. While only inferences can be made about those ancestral mammals, it is possible that extant clades of mammals that diverged early from the ancestral eutherian mammals might still share those rules. Marsupials (or Metatheria) are one such clade: an infraclass that diverged within Mammalia relatively early in mammalian evolution, about 148 million years ago (Mya) (Fig. 1a) [Murphy et al., 2001, 2004; BinindaEmonds et al., 2007], and thus they are the closest living relatives of placental (eutherian) mammals. Marsupialia consists of nearly 350 extant species divided into 4 Australasian and 3 South American orders [Nilsson et al., 2010; Gallus et al., 2015; May-Collado et al., 2015]. While the neuroanatomy, connectivity, neocortical development, and physiology of the brain in some representative marsupial species have been studied [Saunders et al., 1989; Rosa et al., 1999; Ashwell et al., 2008; Wong and Kaas, 2009; Watson et al., 2012], little is known about the cellular composition of marsupial brains and how it compares with other clades, besides a report of a low neuronal density in the neocortex of a single species, the opossum (Didelphis virginiana), in comparison to other mammals [Haug, 1987]. Recently, two more detailed studies were conducted on the cellular composition of another species, the gray short-tailed opossum Monodelphis domestica, during development [Seelke et al., 2013] and across the primary sensory fields of its neocortex [Seelke et al., 2014].

Here we determine the number of cells that compose the brain of a range of marsupial species to examine: (1) whether the neuronal scaling rules shared by extant Afrotheria, Artiodactyla, Glires, Scandentia, and Eulipo- 


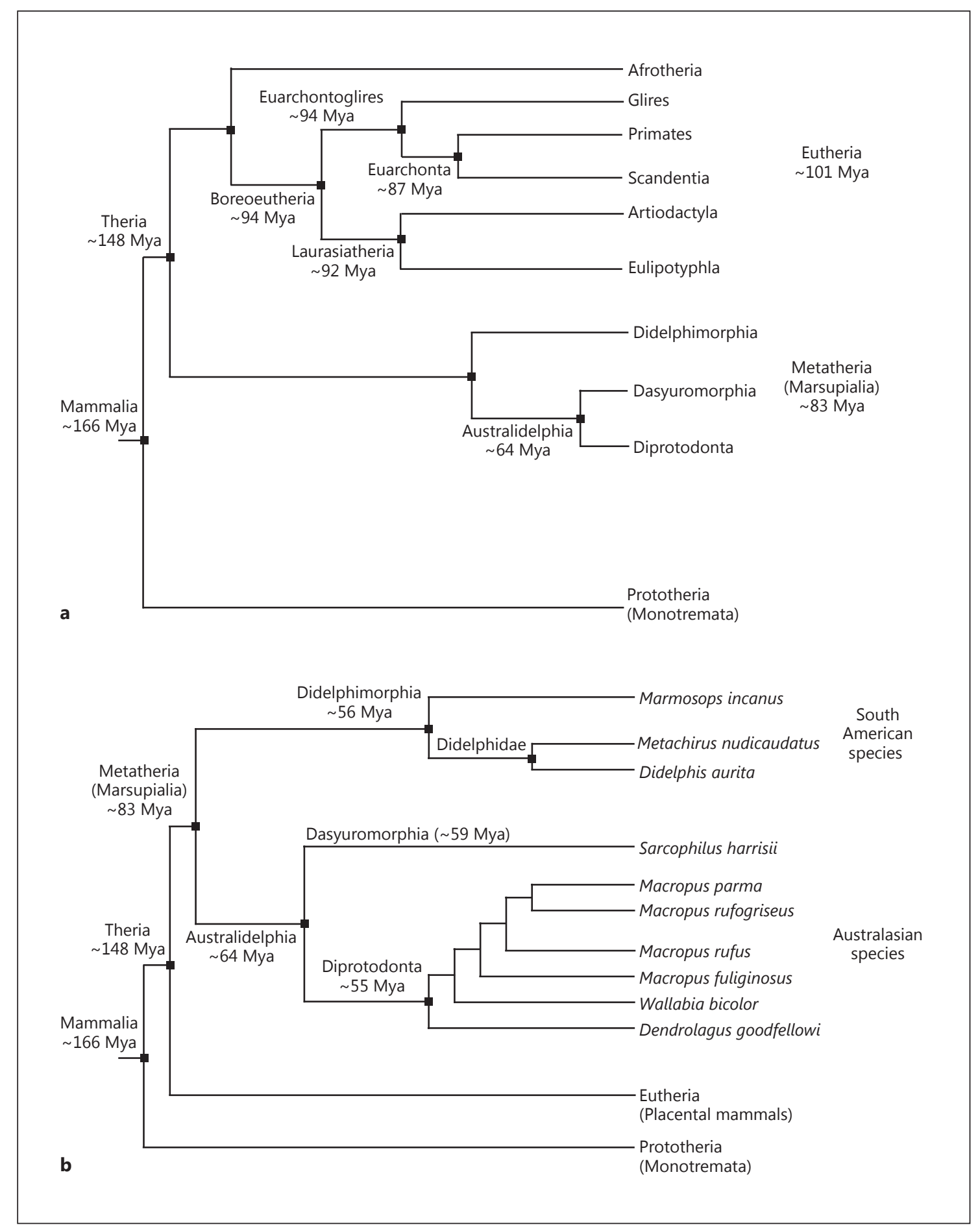

Fig. 1. Phylogenetic relationship of the marsupial species used in this study and their relationship to other mammals. a Phylogenetic relationship across eutherian orders investigated previously and the marsupial orders used in the present study. b Detailed phylogenetic relationship across the marsupial species examined here (both South American and Australasian species). The average times of basal diversification of each clade are in millions of years ago (Mya) and based on data from Murphy et al. [2004] and Binin-
da-Emonds et al. [2007]. Marmosops incanus, gray slender mouse opossum; Metachirus nudicaudatus, brown four-eyed opossum; Didelphis aurita, big-eared opossum; Sarcophilus harrisii, Tasmanian devil; Macropus parma, Parma wallaby; Macropus rufogriseus, Bennett's wallaby; Macropus rufus, red kangaroo; Macropus fuliginosus, Western gray kangaroo; Wallabia bicolor, swamp wallaby; and Dendrolagus goodfellowi, Goodfellow's tree kangaroo. 
typhla and inferred to apply to the most recent common ancestor of all eutherian mammals are also shared by marsupials and (2) whether the putative universality of the nonneuronal composition of the eutherian brain also extends to marsupial brains. We use the isotropic fractionator [Herculano-Houzel and Lent, 2005], a nonstereological method that yields results similar to those obtained with stereology [Herculano-Houzel et al., 2015b], to investigate the cellular composition of the brains of 10 different species of marsupials: 3 belonging to the South American order Didelphimorphia and 7 belonging to the Australasian orders Dasyuromorphia and Diprodonta (Fig. 1b). Our finding that several scaling relationships are not shared across extant marsupial and nonprimate eutherian species, despite their early divergence in mammalian evolutionary history, argues against the common use of the brains of extant Marsupialia as proxies for the ancestral mammalian brain.

\section{Material and Methods}

All collection, dissection, tissue processing, and mathematical procedures were performed as in our previous studies to ensure that all data obtained could be compared directly to those already published for eutherian species [collected in Herculano-Houzel et al., 2015a]. Briefly, brains were either perfused or immersion-fixed with $4 \%$ paraformaldehyde, dissected according to similar criteria (as detailed below), and individual brain structures were subjected to isotropic fractionation. Because cell counts obtained with the isotropic fractionator are independent of tissue volume and the integrity of fine cellular aspects, but rather require simply that the nuclear membrane remain intact, the method of fixation has no expected consequences for the data obtained. That both perfusion and immersion fixation methods were sufficient to make nuclei resistant to fractionation was ascertained by visual inspection of the suspensions, which typically showed no broken nuclei.

\section{Animals}

We examined 1 specimen each of Goodfellow's tree kangaroo (Dendrolagus goodfellowi), Western gray kangaroo (Macropus fuliginosus), red kangaroo (M. rufus), swamp wallaby (Wallabia bicolor), Parma wallaby (M. parma), Bennett's wallaby (M. rufogriseus), Tasmanian devil (Sarcophilus harrisii), big-eared opossum (Didelphis aurita), gray slender mouse opossum (Marmosops incanus), and brown four-eyed opossum (Metachirus nudicaudatus). The Diprotodontia and Dasyuromorphia Australasian specimens ( $n=7 ; 3$ kangaroos: D. goodfellowi, M. fuliginosus, and $M$. rufus; 3 wallabies: W. bicolor, M. parma, and M. rufogriseus; and the Tasmanian devil S. harrisii) came from the Cleveland $\mathrm{Me}$ troparks Zoo (kangaroos and wallabies) and the Copenhagen Zoo (Tasmanian devil), where they died of natural death or from nonneurological diseases. All Australasian specimens were treated and used in accordance with the George Washington University IACUC (clearance No. A117) and the University of the Witwatersrand Animal Ethics Committee (clearance No. 2012/53/01), which parallel those of the NIH for the care and use of animals in scientific experiments. Collections of South American Didelphimorphia marsupials were performed with license number 12685 1/2011 issued by ICMBio (Brazilian Ministry of Environment). Three Didelphimorphia specimens were collected using live traps in Cidade Universitária, Rio de Janeiro ( $D$. aurita), and in Centro Marista São José das Palmeiras, Mendes (M. incanus and M. nudicaudatus), both localities in the State of Rio de Janeiro, Southeastern Brazil. Voucher specimens in the form of skins, skeletons, and tissues are deposited in the Laboratório de Mastozoologia of Universidade Federal do Rio de Janeiro under the numbers WCT 43, 48, and 49. Field procedures and the treatments applied to collect specimens were in accordance with American Society of Mammalogists guidelines for the use of wild mammals in research [Sikes and Gannon, 2011]. All animals were healthy with no obvious pathologies upon veterinary examination, with no visible neuropathologies, and had the typical body mass of adults.

\section{Dissection}

Australasian specimens were collected after natural death and their brains were fixed by immersion in $4 \%$ paraformaldehyde in $0.1 \mathrm{M}$ phosphate buffer ( $\mathrm{PB} ; \mathrm{pH} 7.4)$ and then stored in a solution of $0.1 \%$ sodium azide in phosphate-buffered saline (PBS) under refrigeration. South American animals were euthanized (overdose of ketamine and xylazine at 300 and $30 \mathrm{mg} / \mathrm{kg}$, respectively) and the heart was perfused through the left heart ventricle. Following perfusion, the brains were removed, weighed, and postfixed in $4 \%$ paraformaldehyde in $0.1 \mathrm{M} \mathrm{PB}$ overnight, cryoprotected in $30 \%$ sucrose in $0.1 \mathrm{M} \mathrm{PB}$ at $4^{\circ} \mathrm{C}$, and stored in an antifreeze solution at $-20^{\circ} \mathrm{C}$ until processing [Manger et al., 2009]. The brains were divided into two halves along the midsagittal fissure and one hemisphere of each brain was processed. The $\mathrm{Cb}$ was dissected by cutting the cerebellar peduncles at the surface of the brainstem. To isolate the $\mathrm{Cx}$ (available for all specimens except the red kangaroo, for which only the $\mathrm{Cb}$ and pons+medulla structures were available), the cerebrum was first separated from the brainstem by cutting at a plane anterior to the colliculi and posterior to the thalamus and mammilary bodies of the hypothalamus. The brainstem was divided into pons+medulla and mesencephalon by an axial transection anterior to the basilar pons and posterior to the inferior colliculus. The cerebrum of all Australasian animals and of the big-eared opossum was then cut manually into 2-mm (Australasian specimens) or 1-mm (big-eared opossum) coronal sections in order to allow removal of the ensemble of diencephalon and striatum, removal of the hippocampus ( $\mathrm{Hp})$, and separation of the remaining $\mathrm{Cx}$ into gray and white matter, which had their numbers of cells counted separately. All brain parts used in this study were dissected as shown in online supplementary Figure S1 (see www.karger.com/doi/10.1159/000452856 for all online suppl. material). As in our previous studies, the $\mathrm{Cx}$ includes all structures lateral to the olfactory tract, including the entorhinal cortex, the pyriform cortex, and the amygdala. Where specified in the text, the Hp was also included in the cortex, for consistency with previous studies. The olfactory bulbs (OB), when available, were dissected free of the olfactory tract (and thus include only the bulb proper) and weighed individually. Numbers of cells obtained separately for the pons+medulla, mesencephalon, and diencephalon+striatum were later pooled together and are reported as RoB, for comparison with data obtained previously in other species [HerculanoHouzel et al., 2006, 2007, 2011, 2014b; Azevedo et al., 2009; Sarko 
et al., 2009; Gabi et al., 2010; Kazu et al., 2014; Neves et al., 2014; all collected in Herculano-Houzel et al., 2015a]. For the sake of consistency with our previous studies, and because the OB was not available for all specimens, whole brain values used in the analysis exclude the OB. Since only one hemisphere of each brain was used for this analysis, values reported here are multiplied by 2 to give estimates for the whole brain that can be compared with our previously published data on eutherians. While this practice ignores possible asymmetries between the hemispheres, and also does not address variation across individuals, such asymmetries and intraspecific variations would have only a negligible influence on the results reported here given that, whereas any asymmetries would be of the order of a few percentage points between the hemispheres and the coefficient of variation in number of brain neurons across mouse individuals is below 15\% [Herculano-Houzel et al., 2015c], the present comparison across species spans several orders of magnitude.

\section{Isotropic Fractionation}

Total numbers of cells, neurons, and nonneuronal (other cells) were estimated as described previously using the Isotropic fractionator method [Herculano-Houzel and Lent, 2005]. Briefly, this method turns each dissected brain division into an isotropic suspension of known, defined volume, containing free isolated nuclei. The suspension is made homogeneous (isotropic) by agitation before samples are collected for counting. The total number of nuclei in suspension - and therefore the total number of cells in the original tissue - is estimated by determining the density of nuclei in small aliquots stained with the fluorescent DNA marker DAPI (4',6-diamidino-2-phenylindole dihydrochloride; Invitrogen, USA) under the microscope.

For each structure, at least 4 samples of the nuclear suspension were counted independently, in different chambers of the hemocytometer, to determine the number of nuclei per milliliter of suspension. The reported values for the total number of cells refer to the average number of nuclei per milliliter of the samples taken multiplied by the total volume of the suspension. This consistently yields a variation coefficient of never more than 0.15 across samples from the same structure.

Once the total cell number in a structure is known, the proportion of neurons is determined by immunocytochemical detection of neuronal nuclear antigen (NeuN), expressed in the nuclei of most neuronal cell types and not in nonneuronal cells [Mullen et al., 1992; Gittins and Harrison, 2004] and evolutionarily conserved enough that the same polyclonal antibody stains all nuclei with anatomical characteristics of neurons, and only those, not only in mammals but also in crocodiles [Ngwenya et al., 2016] and birds [Olkowicz et al., 2016]. We used the rabbit polyclonal primary antibody against NeuN that is conjugated with Cy3 (ABN78C3; Millipore) diluted at 1:150 in PBS (0.1 M, pH 7.4). We could verify that all labeled free nuclei had the spherical shape and loose chromatin that are typical of neurons, and were consistently similar among themselves and different from all other nonlabeled nuclei, given that all counts were made manually at the microscope.

We conducted immunohistochemistry on $50-\mu \mathrm{m}$ coronal sections of cerebral cortices from 2 South American (M. incanus and $M$. nudicaudatus) and 2 Australasian species (M. rufogriseus and $S$. harrisii) to confirm that the anti-NeuN antibody labeled all neurons and only neurons in the analyzed species of this study. The same dilution of anti-NeuN antibody used on free nuclei was ap- plied on tissue sections in the presence of goat and bovine sera (5\% normal goat serum and 3\% bovine serum albumin in PBS 0.1 $\mathrm{M} / 0.5 \%$ Triton X-100). Labeled cells have the typical round nuclear and often pyramidal cell body shape of neurons and they have the expected distribution (gray matter of the cortex with high densities in particular layers; hippocampal CA1, CA3, and dentate gyrus; online suppl. Fig. S2 and S3). Labeling in the white matter and striatum is nonspecific and not localized on nuclei.

Estimates of the proportion of NeuN-positive nuclei are considered reliable since the coefficient of variation among animals of the same species is typically below 0.15 . Numbers of neurons reported refer to the product of the number of cells in a structure and the fraction of nuclei that were NeuN positive. Numbers of other (nonneuronal, NeuN-negative) cells are derived by subtraction of the number of neurons from the total number of cells.

\section{Data Analysis}

All statistical analyses and regressions (power laws and linear functions as well as the analysis of residues) were performed in JMP 10.0 (SAS Institute, NC, USA). Regressions to power and linear functions were performed to find the best fit for each distribution. All exponents are reported \pm standard error (SE) and with the corresponding $p$ value. Confidence intervals (95\% CI) are not reported explicitly as they can be easily calculated as exponent \pm 2 SE.

For the comparison with cellular scaling rules reported previously, we used the equations that apply to the average structure size and cellular composition for the species of the groups described earlier: Primata (Otolemur garnettii, Microcebus murinus, Callimico goeldii, Callithrix jacchus, Cebus apella, Saimiri sciureus, Aotus trivirgatus, Macaca mulatta, M. fascicularis, M. radiata, Papio anubis cynocephalus, and Homo sapiens [Azevedo et al., 2009; Gabi et al., 2010]); Glires (Oryctolagus cuniculus, Cynomy sp., Sciurus carolinensis, Mus musculus, Rattus norvegicus, Mesocricetus auratus, Cavia porcellus, Hydrochoerus hydrochaeris, Dasyprocta primnolopha, and Proechimys cayennensis [Herculano-Houzel et al., 2006, 2011]); Eulipotyphla (Sorex fumeus, Blarina brevicauda, Parascalops breweri, Scalopus aquaticus, and Condylura cristata [Sarko et al., 2009]); Afrotheria (Loxodonta africana, Procavia capensis, Dendrohyrax dorsalis, Amblysomus hottentotus, Elephantulus myurus, and Petrodomus tetradactylus [Herculano-Houzel et al., 2014b; Neves et al., 2014]); and Artiodactyla (Giraffa camelopardalis, Damaliscus dorcas phillipsi, Antidorcas marsupialis, Tragelaphus strepsiceros, and Sus scrofa domesticus [Kazu et al., 2014]). The complete dataset is available in the paper by Herculano-Houzel et al. [2015a], which also explains the rationale for excluding a few species from group analyses. Briefly, all analyses excluded the naked mole rat, the only fossorial animal in our sample, which is an outlier among Glires [Herculano-Houzel et al., 2011]. The giraffe was excluded from analyses of scaling of total brain mass as well as cortical mass and neuronal density since the specimen available was still a juvenile [Kazu et al., 2014]; the pig, a domesticated species, was excluded from analyses involving body mass [Kazu et al., 2014], and the elephant was excluded from analyses involving the $\mathrm{Cb}$ and total brain mass and number of neurons [Herculano-Houzel et al., 2014b]. Because we found the marsupial data points to overlap with the distributions for glires, afrotherians, artiodactyls, and eulipotyphla, we also tested their alignment with the power function that applies jointly to these groups by analyzing the residuals and applying a Wilcoxon statistical test. 
Fig. 2. Marsupial brains gain mass and neurons similarly to Afrotheria, Glires, Scandentia and Eulipotyphla but not primates. Total brain mass (a, excluding the olfactory bulb) and total number of neurons in the brain (b, excluding the olfactory bulb) scale with body mass similarly across marsupials (black data points) and nonprimate, nonartiodactyl species (light gray). Only the power functions for Afrotheria (minus the elephant), Glires, Scandentia, and Eulipotyphla are plotted, along with the 95\% confidence interval (dotted lines). Exponents are $0.693 \pm 0.043\left(\mathbf{a}, \mathrm{M}_{\mathrm{BR}} \times \mathrm{M}_{\mathrm{BD}}\right)$ and $0.439 \pm 0.038\left(\mathbf{b}, \mathrm{N}_{\mathrm{BR}} \times \mathrm{M}_{\mathrm{BD}}\right)$. Species are shown in shades of gray as displayed in $\mathbf{a}$, with marsupials in black, primates in dark gray, artiodactyls in medium gray, and nonprimate, nonartiodactyl species in light gray. Values are exponents \pm SE. Data are from Herculano-Houzel et al. [2006, 2007, 2011] Azevedo et al. [2009], Sarko et al. [2009], Gabi et al. [2010], Kazu et al. [2014], and Neves et al. [2014]. $\mathrm{M}_{\mathrm{BR}}$, brain mass; $\mathrm{M}_{\mathrm{BD}}$, body mass; $\mathrm{N}_{\mathrm{BR}}$, brain neurons.
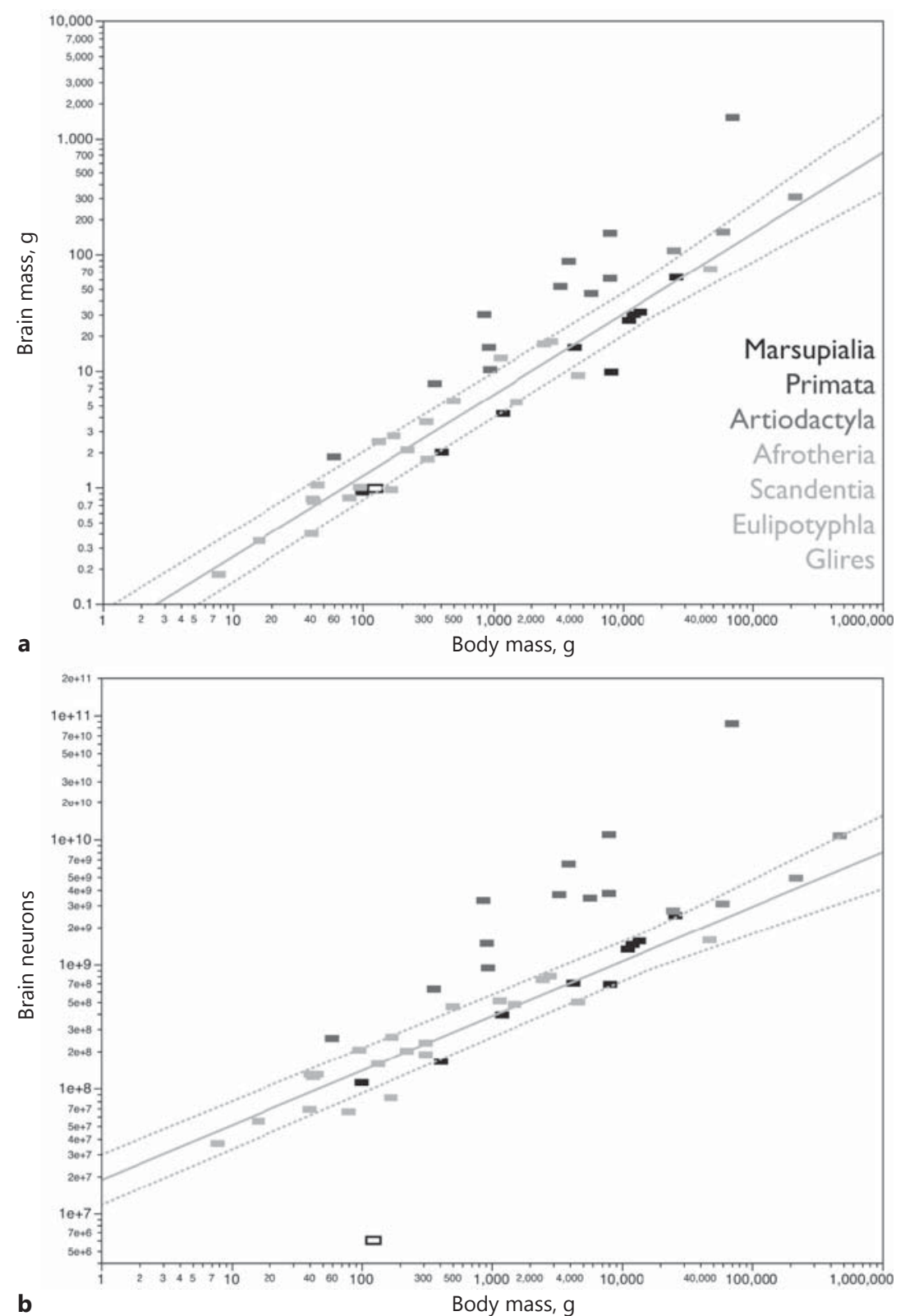

\section{Results}

Across the 10 marsupial species examined (Fig. 1b), body mass $\left(\mathrm{M}_{\mathrm{BD}}\right)$ varies 258 -fold, from $100 \mathrm{~g}$ in the gray slender mouse opossum to $25,855 \mathrm{~g}$ in the Western gray kangaroo, while brain mass $\left(\mathrm{M}_{\mathrm{BR}}\right)$ varies 69 -fold (between $0.910 \mathrm{~g}$ in the gray slender mouse opossum and $62.724 \mathrm{~g}$ in the Western gray kangaroo), and the total number of brain neurons varies only 22 -fold (online suppl. Table S1; Fig. 2). $\mathrm{M}_{\mathrm{BR}}$ varies as a power function of $\mathrm{M}_{\mathrm{BD}}$ with an exponent of $0.742 \pm 0.061(p<0.0001)$, with a $95 \%$ CI that overlaps with that for the joint exponent of $0.693 \pm 0.043(p<0.0001$; Fig. 2a) previously found for Afrotheria, Glires, Scandentia, and Eulipotyphla [Herculano-Houzel et al., 2006, 2011; Sarko et al., 2009; Neves et al., 2014] but does not overlap with 
Fig. 3. Marsupials fit the nonneuronal scaling rules for all brain structures and mammalian clades. Marsupial species fit the relationship between structure mass and number of nonneuronal (other, O) cells for each brain structure (cerebral cortex, cerebellum, and rest of brain) (a) and the relationship between the density of other cells in the structures and the number of other cells in the structure found across all eutherian species examined (b). The function plotted in a applies to the ensemble of nonmarsupial species with exponent $1.052 \pm$ 0.017 . Species are shown in shades of gray as displayed in a. Values are exponents \pm SE. Data are from Herculano-Houzel et al. [2006, 2007, 2011, 2014b], Azevedo et al. [2009], Sarko et al. [2009], Gabi et al. [2010], Kazu et al. [2014], and Neves et al. [2014].

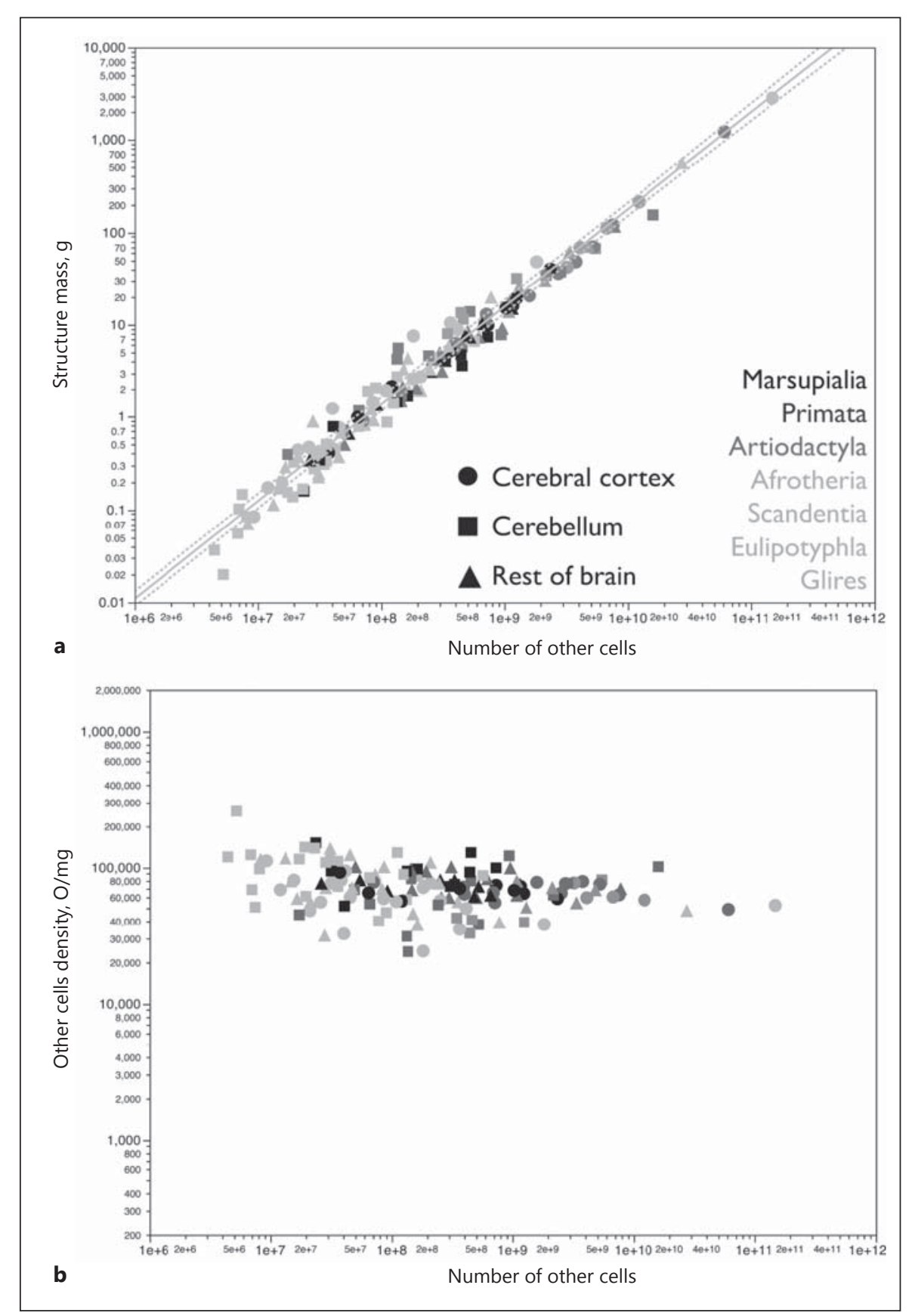

those found for Primata and Artiodactyla [HerculanoHouzel et al., 2007; Azevedo et al., 2009; Gabi et al., 2010; Kazu et al., 2014]. Inclusion of the gray shorttailed opossum (M. domestica; open symbol in Fig. 2a [Seelke et al., 2013]), the only data point found in the literature, hardly changes the relationship between $\mathrm{M}_{\mathrm{BR}}$ and $\mathrm{M}_{\mathrm{BD}}$ (exponent $0.735 \pm 0.049, p<0.0001$ ). Most marsupial species are included in the $95 \% \mathrm{CI}$ of the joint distribution for Afrotheria, Glires, Scandentia, and Eulipotyphla (Fig. 2a).

The total number of brain neurons $\left(\mathrm{N}_{\mathrm{BR}}\right)$ increases across marsupial species in our dataset as a power function of $\mathrm{M}_{\mathrm{BD}}$ with a smaller exponent of $0.554 \pm 0.041(p<$ 0.0001 ) with a distribution that overlaps with that found previously for Glires, Eulipotyphla, Scandentia, and Afrotheria (Fig. 2b), but not for Primata and Artiodactyla. 
Remarkably, the total number of neurons estimated for Monodelphis by [Seelke et al., 2013] using the same method is grossly smaller than expected for its body mass (open symbol in Fig. 2b). The relationships between numbers of neurons and mass across brain structures (online suppl. Fig. S4) also show that Monodelphis has grossly smaller numbers of neurons than expected for the mass of the different brain structures. This suggests that either Monodelphis is an outlier among marsupials or its reported cellular composition is an underestimate. We thus decided to exclude data for this species from our further analyses.

\section{Relative Distribution of Mass and Neurons}

The $\mathrm{Cx}$ (including the $\mathrm{Hp}$ ) varies 98 -fold in mass but only 18 -fold in number of neurons across the species in our sample (online suppl. Table S1). The Cx represents $55.2 \pm 2.4 \%$ of brain mass across species but holds only $15.9 \pm 1.0 \%$ of all brain neurons. Larger brains have relatively larger cortices (Spearman's correlation, $\rho=0.833$, $p=0.0053$ ), but larger brains do not have a greater percentage of their neurons located in the Cx (Spearman, $p=$ 0.7324 ), such that relatively larger cortices do not have proportionally more neurons (Spearman, $p=0.3317$ ). The relatively larger $\mathrm{Cx}$ of larger marsupial brains accompanies a relatively smaller $\mathrm{Cb}$ and $\mathrm{RoB}$ (Spearman, $\rho=-0.683$ and $\rho=-0.733$, respectively; $p=0.0424$ and $p=0.0246$ ). Again, relatively smaller cerebella and RoB do not have a smaller percentage of all brain neurons (Spearman, $p=0.3085$ and $p=0.3558$, respectively). While the $\mathrm{Cb}$ and RoB represent $14.6 \pm 0.8$ and $30.2 \pm 1.6 \%$ of the brain mass, respectively, the $\mathrm{Cb}$ houses $80.0 \pm 1.1 \%$ of all $\mathrm{N}_{\mathrm{BR}}$, and the remaining $4.0 \pm 0.7 \%$ of $\mathrm{N}_{\mathrm{BR}}$ are located in the RoB.

Within the $\mathrm{Cx}$, the $\mathrm{Hp}$ represents on average $11.9 \pm$ $1.2 \%$ of the cortical mass and holds on average $10.7 \pm$ $1.8 \%$ of all cortical neurons. While larger cortices contain relatively smaller hippocampi (Spearman, $\rho=-0.9000$, $p=0.0009$ ), the correlation between cortical mass and the percentage of cortical neurons located in the Hp does not reach significance $(p=0.0992)$.

\section{Nonneuronal Scaling Rules}

All brain structures in our dataset $(\mathrm{Cx}, \mathrm{Cb}$, and $\mathrm{RoB})$ vary in mass across marsupial species as a single power function of the number of nonneuronal cells in the structure (Fig. 3a; exponent $1.049 \pm 0.034, p<0.0001$ ) that overlaps with the distribution for all other mammalian species analyzed so far (exponent $1.052 \pm 0.017, p<$ $0.0001)$. In line with the near linearity of scaling of brain structure mass with numbers of nonneuronal cells, there is very little and nonsystematic variation in the density of nonneuronal cells in all marsupial brain structures (Fig. 3b). The joint exponent for all Theria $(1.051 \pm 0.015$, $p<0.0001$ ) indicates that brain structures of a similar size are composed of similar numbers of nonneuronal cells across different modern Theria clades, including marsupials.

As observed for other therian groups, neuronal density varies considerably more than nonneuronal cell density across marsupial brain structures (online suppl. Fig. S5A, B) - while neuronal densities span over 2 orders of magnitude, nonneuronal cell densities vary only 3 -fold (online suppl. Table S1). Across marsupial species, no brain structure exhibits a significant correlation between nonneuronal cell density and structure mass $(\mathrm{Cx}, p=$ 0.2246 ; $\mathrm{Cb}, p=0.4868$; and $\mathrm{RoB}, p=0.2660$ ). The ratio between numbers of nonneuronal cells and neurons in each structure $(\mathrm{O} / \mathrm{N})$ varies between 0.122 (in the bigeared opossum $\mathrm{Cb}$ ) and 20.583 (in the swamp wallaby RoB) across structures and species in marsupials (online suppl. Table S1), with no single evident relationship with structure mass (online suppl. Fig. S6A). In contrast, and as found in other mammalian species, $\mathrm{O} / \mathrm{N}$ varies as a common power function of neuronal density across all marsupial structures and species with an exponent of $-0.925 \pm 0.022(p<0.0001)$, in a distribution that overlaps with the variation of $\mathrm{O} / \mathrm{N}$ as a function of neuronal density across nonmarsupial species (exponent $-0.939 \pm$ $0.022, p<0.0001$, gray plot in online suppl. Fig. S6B; joint exponent for all therian species $-0.938 \pm 0.019, p<$ 0.0001).

\section{Neuronal Scaling Rules}

Across the marsupial species studied, $\mathrm{M}_{\mathrm{BR}}$ varies as a power function of the total number of brain neurons with an exponent of $1.338 \pm 0.056$ ( $p<0.0001$; Fig. 4a), significantly above unity, which indicates that the brain as a whole gains mass faster than it gains neurons. Marsupial data points fall well within the $95 \% \mathrm{CI}$ of the scaling relationship that applies jointly to Eulipotyphla, Afrotheria (minus the elephant, a major outlier [Herculano-Houzel et al., 2014a]), Glires, and Artiodactyla (minus the giraffe, a juvenile [Herculano-Houzel et al., 2015a]), of exponent $1.496 \pm 0.052(p<0.0001)$, shown in Figure 4a. The neuronal scaling rules found for marsupials are listed in online supplementary Table S2.

The marsupial $\mathrm{Cx}$ in particular also conforms to the neuronal scaling rules that apply to the ensemble of Afrotheria, Glires, Eulipotyphla, and Artiodactyla (minus the giraffe juvenile [Herculano-Houzel et al., 2015a]). 
The $\mathrm{M}_{\mathrm{CX}}$ scales in marsupials with the number of neurons in the $\mathrm{Cx}\left(\mathrm{N}_{\mathrm{CX}}\right)$ raised to an exponent of $1.329 \pm$ 0.097 ( $p<0.0001$; Fig. 4b). While this falls below the $95 \%$ CI for the exponent that applies to Afrotheria, Glires, Eulipotyphla, and Artiodactyla together $(1.631 \pm 0.040$; $p<0.0001$, giraffe juvenile excluded), marsupial data points fall well within the $95 \%$ CI calculated for other nonprimate, nonscandentian clades (Fig. 4b). Analysis of the residuals for marsupial species calculated for the $\mathrm{M}_{\mathrm{CX}} \times \mathrm{N}_{\mathrm{CX}}$ relationship that applies to nonprimate, nonscandentian clades shows that these residuals are not systematically positive or negative and therefore as a group are not significantly different from zero (Wilcoxon, $p=0.3291$ ). The marsupial $\mathrm{Cx}$ thus has the mass expected for its number of neurons in conformity with other nonprimate, nonscandentian species examined previously.

The conformity of the marsupial $\mathrm{Cx}$ with the nonprimate scaling rule is supported by the finding that the neuronal density in the $\mathrm{Cx}\left(\mathrm{DN}_{\mathrm{CX}}\right)$ decreases uniformly across marsupial species as the $\mathrm{Cx}$ gains neurons with an exponent of $-0.329 \pm 0.097(p=0.0117)$. While this exponent falls above the $95 \% \mathrm{CI}$ for the exponent that applies to nonprimate, nonscandentian species $(-0.631 \pm 0.040$; $p<0.0001$, giraffe juvenile excluded), all data points for marsupial species fall within the $95 \%$ CI for those species (Fig. 4e). Because neuronal density varies with the inverse of average neuronal cell size [Mota and Herculano-Houzel, 2014], these data suggest that cortical expansion in all nonprimate, nonscandentian Theria examined so far, including marsupials, occurred with an addition of neurons whose average size increased as a common function of $\mathrm{N}_{\mathrm{CX}}$.

The $\mathrm{Cb}$ scales in mass $\left(\mathrm{M}_{\mathrm{CB}}\right)$ across marsupial species as a power function of its number of neurons $\left(\mathrm{N}_{\mathrm{CB}}\right)$ that has an exponent of $1.186 \pm 0.037(p<0.0001)$, below the $95 \%$ $\mathrm{CI}$ for the exponent found previously for Afrotheria, Artiodactyla, and Glires together $(1.283 \pm 0.035, p<0.0001$, excluding the elephant, a major outlier [Herculano-Houzel et al., 2014a]), and above the 95\% CI for the exponents for Primata and Scandentia $(0.983 \pm 0.032, p>0.0001)$ and for Eulipotyphla (1.028 $\pm 0.084, p=0.0012)$ (Fig. 4c). However, most marsupial data points for the $\mathrm{Cb}$ fall below the 95\% CI for Afrotheria, Artiodactyla, and Glires (Fig. 4c). Indeed, the analysis of the residuals for marsupial species calculated for the $\mathrm{M}_{\mathrm{CB}} \times \mathrm{N}_{\mathrm{CB}}$ relationship that applies to nonprimate, noneulipotyphlan clades shows that 9 of 10 marsupial species have negative residuals in comparison to Afrotheria, Artiodactyla, and Glires (with the exception of the brown four-eyed opossum, positive residual 2.5; Wilcoxon, $p=0.0045$ ), and positive residuals for 9 of 10 species in comparison to Eulipotyphla and Primata $(p=0.0027$ and $p=0.0009$, respectively). Thus, for a same $\mathrm{M}_{\mathrm{CB}}$, marsupials have fewer $\mathrm{N}_{\mathrm{CB}}$ than primates and eulipotyphlans but more $\mathrm{N}_{\mathrm{CB}}$ than afrotherians, glires, or artiodactyls.

In line with this marsupial-specific scaling of the $\mathrm{Cb}$, the cerebellar neuronal density $\left(\mathrm{DN}_{\mathrm{CB}}\right)$ of several marsupial species is larger than the $\mathrm{DN}_{\mathrm{CB}}$ of afrotherians, glires, and artiodactyls of similar $\mathrm{N}_{\mathrm{CB}}$ but smaller than the $\mathrm{DN}_{\mathrm{CB}}$ of primates or eulipotyphlans (Fig. $4 \mathrm{f}$ ). $\mathrm{DN}_{\mathrm{CB}}$ decreases at a significantly slower rate with increasing $\mathrm{N}_{C B}$ across marsupials (exponent $-0.186 \pm 0.037, p=0.0010$; Fig. 4f)
Fig. 4. Marsupials share neuronal scaling rules for the cerebral cortex but not for the cerebellum and rest of brain with other nonprimate mammals. The average brain structure mass (a-d) or neuronal density $(\mathbf{e}-\mathbf{g})$ for each species are plotted as a function of its total number of neurons. a Whole brain (excluding the olfactory bulb). b, e Cerebral cortex (including the hippocampus). c, f Cerebellum. $\mathbf{d}, \mathbf{g}$ Rest of brain. a The neuronal scaling rule that applies to the whole brain of nonprimate, nonscandentian mammals (plotted fit, which excludes the elephant and the giraffe; exponent $1.496 \pm 0.052, p=0.0001$ ) includes marsupial species. b Marsupials overlap with the power function relating $\mathrm{M}_{\mathrm{CX}}$ (including the hippocampus) and $\mathrm{N}_{\mathrm{CX}}$ for Afrotheria, Glires, Eulipotyphla, and Artiodactyla together (plotted fit, minus the giraffe; exponent $1.631 \pm 0.040)$, and the power function that applies to marsupials (exponent $1.329 \pm 0.097$, not plotted) also includes these species (joint exponent $1.587 \pm 0.040$, not shown) but excludes primates. c The neuronal scaling rule that applies to the cerebellum of Afrotheria (excluding the elephant), Artiodactyla, and Glires (expo- nent $1.283 \pm 0.035, p<0.0001)$ excludes some marsupials. $\mathbf{d}$ The neuronal scaling rule that applies to the rest of brain of Afrotheria, Artiodactyla, Eulipotyphla, and Glires (exponent 1.847 \pm 0.099 ) excludes some marsupial species. e The marsupial cerebral cortex fits the scaling of neuronal density as a power function of exponent $-0.631 \pm 0.040$ that applies to nonprimate, nonscandentian species (plotted fit). $\mathbf{f}$ The cerebellum of most marsupial species examined has larger neuronal densities than those found in nonprimate, noneulipotyphlan species, although most fall within the 95\% CI for the power function (exponent $-0.283 \pm 0.035, p<0.0001$ ). $\mathbf{g}$ The neuronal density in the rest of brain of most marsupial species examined also falls below the function plotted for nonprimate, nonscandentian species (exponent $-0.845 \pm 0.099$ ), although still within the $95 \%$ CI. Species are shown in shades of gray as displayed in $\mathbf{a}$. Values are exponents \pm SE. Data are from Herculano-Houzel et al. [2006, 2007, 2011, 2014b], Azevedo et al. [2009], Sarko et al. [2009], Gabi et al. [2010], Kazu et al. [2014], and Neves et al. [2014].

(For figure see next page.)
Dos Santos et al. 


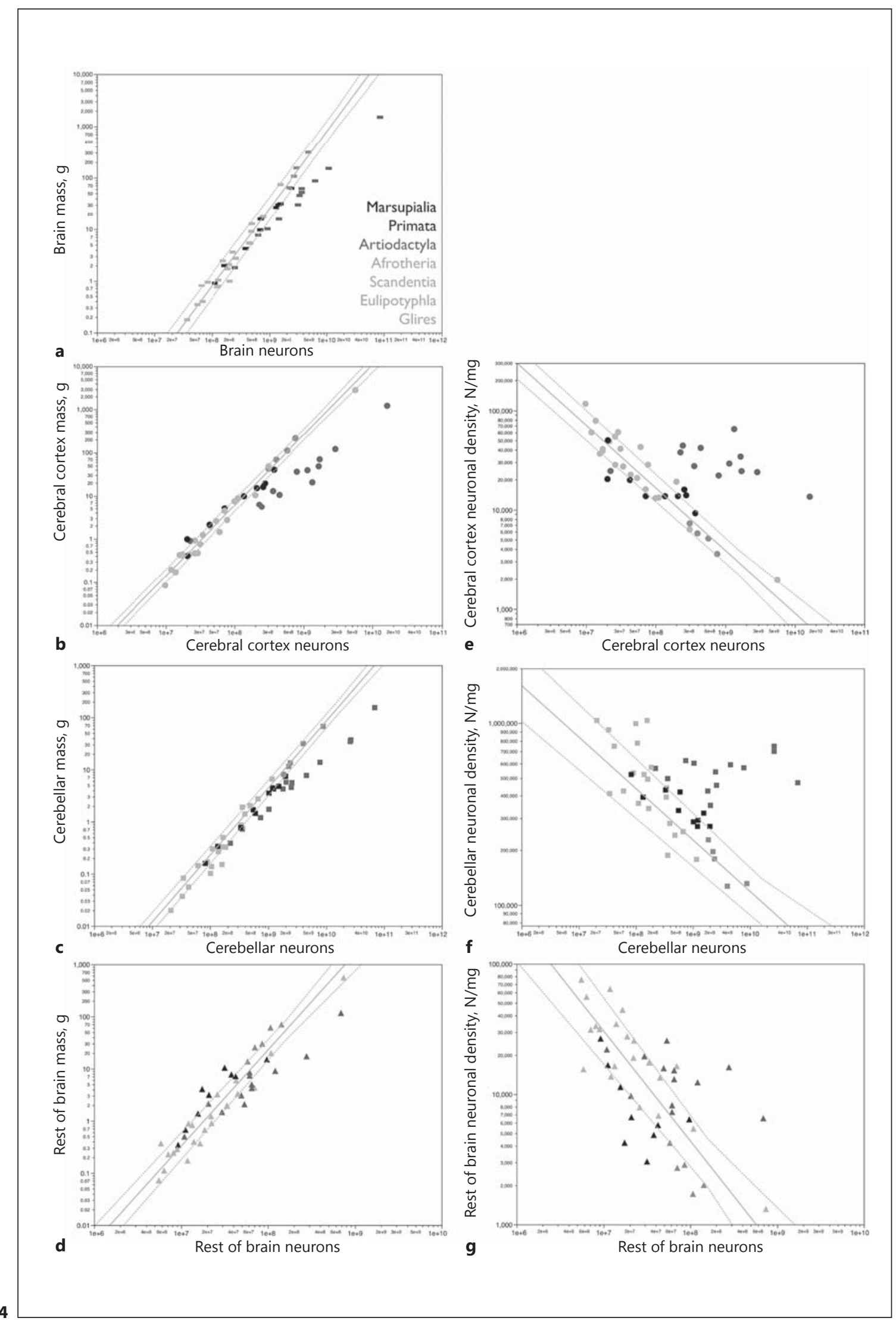


than across afrotherians, glires, and artiodactyls (exponent $-0.283 \pm 0.035, p<0.0001$, excluding the elephant).

The mass of the $\operatorname{RoB}\left(\mathrm{M}_{\mathrm{ROB}}\right)$ scales with the number of neurons in the marsupial $\operatorname{RoB}\left(\mathrm{N}_{\mathrm{ROB}}\right)$ as a power function of exponent $1.598 \pm 0.274(p=0.0006)$, which lies below the $95 \%$ CI for the exponent that applies for all nonprimate, nonscandentian mammals (exponent $1.847 \pm 0.099$, $p<0.0001$; Fig. $4 \mathrm{~d}$ ) but above the $95 \%$ CI for the exponent found for primates and scandentians $(1.226 \pm 0.110, p<$ $0.0001)$. Some marsupial data points fall above the $95 \%$ CI plotted for other nonprimate, nonscandentian species (Fig. 4d), and an analysis of the residuals for marsupial species calculated for the $\mathrm{M}_{\mathrm{ROB}} \times \mathrm{N}_{\mathrm{ROB}}$ relationship that applies to nonprimate, nonscandentian clades shows that these residuals are positive for 9 of 10 species (Wilcoxon, $p=0.0108$ ). Similarly, the same 9 of 10 marsupial species have positive residuals in comparison to Primata and Scandentia (Wilcoxon, $p=0.0062$ ). Thus, for a same $\mathrm{N}_{\mathrm{ROB}}$, marsupials have a larger $\mathrm{M}_{\mathrm{ROB}}$ than primate and nonprimate eutherians.

Neuronal densities in the marsupial $\operatorname{RoB}\left(\mathrm{DN}_{\mathrm{ROB}}\right)$ decrease as a power function of numbers of neurons in the structure in a manner that approaches statistical significance $(p=0.0654)$. $\mathrm{DN}_{\mathrm{ROB}}$ in most marsupial species examined are lower than the $\mathrm{DN}_{\mathrm{ROB}}$ of both primates and nonprimates of similar $\mathrm{N}_{\mathrm{ROB}}$ (Fig. $4 \mathrm{~g}$ ), which indicates that neurons in the RoB of marsupials are larger than in the RoB of eutherians with a similar $\mathrm{N}_{\mathrm{ROB}}$. Importantly, we find that marsupials share with all other eutherians examined the relationship between $\mathrm{DN}_{\mathrm{ROB}}$ and $\mathrm{M}_{\mathrm{BD}}$, with most marsupial species falling within the $95 \% \mathrm{CI}$ of the power function of exponent $-0.302 \pm$ $0.020(p<0.0001)$ that applies to eutherians (online suppl. Fig. S7A), which supports the suggestion that the

Fig. 5. Relative increase in numbers of neurons in the cerebral cortex and cerebellum of Australasian marsupials, with a shared scaling of numbers of neurons across these structures. a Scaling of $\mathrm{N}_{\mathrm{CB}}$ as a function of $\mathrm{N}_{\mathrm{CX}}$ varies in a similar way for all theria with exponents near linearity: $0.931 \pm 0.076$ in marsupials, $0.867 \pm 0.108$ in primates, $0.923 \pm 0.110$ in artiodactyls, and $1.063 \pm 0.111$ in afrotherians, glires, and scandentian together (plotted line). b The $\mathrm{N}_{C X}$ in American marsupials matches the expected for $\mathrm{N}_{\mathrm{ROB}}$ (closed black symbols), while Australasian marsupial species have much higher $\mathrm{N}_{\mathrm{CX}}$ than expected for their $\mathrm{N}_{\mathrm{ROB}}$ (open symbols). c Accordingly, $\mathrm{N}_{\mathrm{CX}} / \mathrm{N}_{\mathrm{ROB}}$ is higher in Australasian marsupials $(6.2 \pm 0.6)$ than in South American marsupials $(2.2 \pm 0.3)$, making the latter comparable to all nonprimate, nonartiodactyla theria and the former comparable to Artiodactyla $\left(\mathrm{N}_{\mathrm{CX}} / \mathrm{N}_{\mathrm{ROB}}=7.3 \pm 1.2\right)$. d $\mathrm{N}_{\mathrm{CB}}$ varies as a power function of $\mathrm{N}_{\mathrm{ROB}}$ of exponent $1.334 \pm 0.212$ average size of neurons in the RoB scales with body length, that is, $\mathrm{M}_{\mathrm{BD}}{ }^{1 / 3}$ [Herculano-Houzel et al., 2015a]. The scaling of $\mathrm{DN}_{\mathrm{ROB}}$ (that is, the inverse of average neuronal cell mass) in conformity with body mass but not with $\mathrm{N}_{\mathrm{ROB}}$ indicates that marsupials must have a different relationship between $\mathrm{N}_{\mathrm{ROB}}$ and $\mathrm{M}_{\mathrm{BD}}$ compared to other species.

Indeed, we find that marsupials gain neurons in the RoB with increasing body mass more slowly than primate species (marsupials, exponent $0.349 \pm 0.070, p=0.0017$; primates, exponent $0.525 \pm 0.089, p=0.0002$ ) but at a rate similar to that of nonprimate, nonscandentian species (exponent $0.332 \pm 0.022, p<0.0001$; online suppl. Fig. S7B). However, for a similar body mass, marsupials have fewer neurons in the RoB than nonprimate eutherians (online suppl. Fig. S7B). In contrast, marsupials gain neurons in the $\mathrm{Cx}$ and $\mathrm{Cb}$ with increasing body mass at the same rate that applies to other nonprimate species and share with these similar numbers of neurons for similar body masses (online suppl. Fig. S7C, D).

The OB was only available for the 3 South American species and the Tasmanian devil. $\mathrm{OB}$ mass $\left(\mathrm{M}_{\mathrm{OB}}\right)$ scales across these species as a power function of its number of neurons $\left(\mathrm{N}_{\mathrm{OB}}\right)$ of exponent $0.833 \pm 0.167(p=0.0380)$, with a $95 \%$ CI that includes unity, and the data are indeed best fitted with a linear function $(p=0.0126)$. Marsupials have an $\mathrm{OB}$ with a range of variation of $\mathrm{M}_{\mathrm{OB}}$ and $\mathrm{N}_{\mathrm{OB}}$ that overlaps with that for Glires and Afrotheria, with a larger $\mathrm{M}_{\mathrm{OB}}$ than eulipotyphlans with a similar $\mathrm{N}_{\mathrm{OB}}$ (online suppl. Fig. S8A).

The mass of the $\mathrm{Hp}\left(\mathrm{M}_{\mathrm{HP}}\right)$ scales across marsupials as a power function of its number of neurons of exponent $1.388 \pm 0.264(p=0.0012)$, with a $95 \%$ CI that overlaps with those found for Eulipotyphla $(1.054 \pm 0.422, p=$

in marsupials, $1.315 \pm 0.112$ in Primata, $1.737 \pm 0.305$ in Artiodactyla, and $1.169 \pm 0.116$ in Afrotheria (minus the elephant), Glires, Eulipotyphla and Scandentia together (plotted line). e $\mathrm{N}_{\mathrm{CB}} / \mathrm{N}_{\mathrm{ROB}}$ is higher in Australasian marsupials $(29.2 \pm 2.5)$ than in South American marsupials $(14.0 \pm 3.7)$, making the former comparable to Artiodactyla and Primata $\left(\mathrm{N}_{\mathrm{CB}} / \mathrm{N}_{\mathrm{ROB}}=38.3 \pm 6.2\right.$ and $35.9 \pm 7.0$, respectively) and the latter significantly different from its Australasian counterpart but also from Afrotheria, Glires, Eulipotyphla and Scandentia together ( $p=0.0112$ and $p=0.0423$, respectively). Species are shown in shades of gray as displayed in a. Australasian marsupials (open symbols) and South American marsupials (closed symbols). Values are exponents \pm SE. Data are from Herculano-Houzel et al. [2006, 2007, 2011, 2014b], Azevedo et al. [2009], Sarko et al. [2009], Gabi et al. [2010], Kazu et al. [2014], and Neves et al. [2014].

(For figure see next page.) DOI: $10.1159 / 000452856$
Dos Santos et al. 
0.0879 ) and for Afrotheria and Artiodactyla together $(1.707 \pm 0.258, p<0.0001$; online suppl. Fig. S8B). Additionally, the $\mathrm{Hp}$ of the marsupial species examined falls within the $95 \% \mathrm{CI}$ for the $\mathrm{M}_{\mathrm{HP}} \times \mathrm{N}_{\mathrm{HP}}$ relationship found for Afrotheria and Artiodactyla, suggesting that the marsupial $\mathrm{Hp}$, like the marsupial $\mathrm{Cx}$ as a whole, shares the same neuronal scaling rule that applies to Afrotheria and Artiodactyla (online suppl. Fig. S8B).

\section{Correlations across Structures}

While the marsupial $\mathrm{Cx}$ shares its neuronal scaling rules with other nonprimate mammals and the marsupial $\mathrm{Cb}$ does not, we find that numbers of neurons in the two structures scale with respect to each other across marsupial species, as they do across all other mammalian groups [Herculano-Houzel et al., 2014a]. This is a linear relationship $\left(r^{2}=0.949, p<0.0001\right)$ or a power function

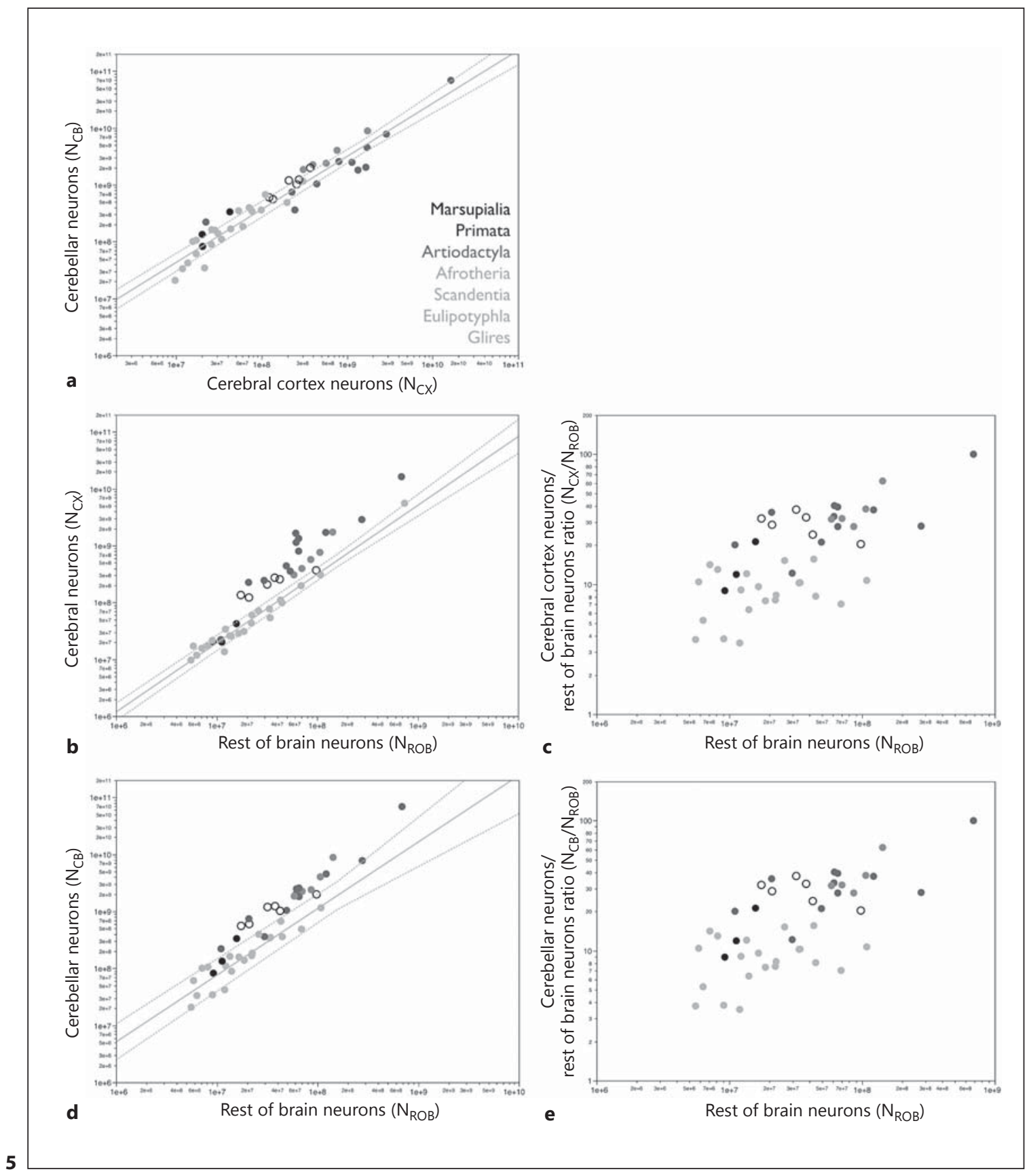


of exponent $0.931 \pm 0.076(p<0.0001)$, indistinguishable from linearity (Fig. 5a). Importantly, all marsupial species fall well within the 95\% CI calculated for other species (excluding the elephant, a major outlier [HerculanoHouzel et al., 2014b]). Thus, marsupials share with eutherians the proportionality between numbers of neurons in the $\mathrm{Cb}$ and in the $\mathrm{Cx}$ of about 4:1 [Herculano-Houzel et al., 2014a].

While the common scaling of numbers of neurons across the $\mathrm{Cb}$ and the $\mathrm{Cx}$ is shared by South American and Australasian marsupial species alike (Fig. 5a), we found that the two groups of marsupials differ markedly in the distribution of neurons across the $\mathrm{Cx} / \mathrm{Cb}$ and the RoB. While South American marsupials share with afrotherians, glires, and eulipotyphlans the relationship between $\mathrm{N}_{\mathrm{CX}}$ and $\mathrm{N}_{\mathrm{ROB}}$ (closed symbols in Fig. 5b), the Cx of Australasian marsupial species has more neurons than any afrotherian, glire, scandentian, or eulipotyphlan with a similar $\mathrm{N}_{\mathrm{ROB}}$ (open symbols in Fig. 5b), approaching values of $\mathrm{N}_{\mathrm{CX}}$ found in primates and artiodactyls of a similar $\mathrm{N}_{\mathrm{ROB}}$. Indeed, the average ratio $\mathrm{N}_{\mathrm{CX}} / \mathrm{N}_{\mathrm{ROB}}$ of $6.2 \pm 0.6$ in Australasian marsupials is not significantly different from the ratio found in artiodactyls ( $7.3 \pm 1.2$; Wilcoxon, $p=0.9273$; Fig. $5 c$ ). In contrast, South American marsupial species have an $\mathrm{N}_{\mathrm{CX}} / \mathrm{N}_{\mathrm{ROB}}$ ratio of only $2.2 \pm 0.3$, similar to that of afrotherian, glire, scandentian, and eulipotyphlan species (Wilcoxon, $p=1.0000$; Fig. 5c).

Similarly, and in line with the coordinated addition of neurons to the $\mathrm{Cx}$ and $\mathrm{Cb}$, Australasian marsupial $\mathrm{Cb}$ have more neurons than any afrotherian, glire, and eulipotyphlan $\mathrm{Cb}$ with a similar $\mathrm{N}_{\mathrm{ROB}}$. Marsupials gain $\mathrm{N}_{\mathrm{CB}}$ as a function of $\mathrm{N}_{\mathrm{ROB}}$ with an exponent of $1.334 \pm 0.212$ $(p=0.0004)$ similar to the exponents of both primates and artiodactyls $(1.315 \pm 0.112, p<0.0001$, and $1.737 \pm 0.304$, $p=0.0107$, respectively; Fig. $5 \mathrm{~d}$ ). The average ratio $\mathrm{N}_{\mathrm{CB}} / \mathrm{N}_{\mathrm{ROB}}$ of $29.2 \pm 2.5$ in Australasian marsupials is not significantly different from the ratio found in both primates (35.9 \pm 7.0 ; Wilcoxon, $p=0.6511)$ and artiodactyls ( $38.3 \pm 6.2$; Wilcoxon, $p=0.4113$ ), but it is close to being significantly higher than in South American marsupials (14.0 \pm 3.7 ; Wilcoxon, $p=0.0528$; Fig. 5e).

\section{Discussion}

Here we show that both Australasian and South American marsupial species share with eutherians a similar scaling relationship between brain structure mass and the number of nonneuronal cells in the structure. The shared relationship indicates that these nonneuronal scaling rules are ancestral and likely shared though common descent in all Theria, dating back to at least 148 Mya, prior the divergence of the Metatheria [Murphy et al., 2001, 2004; Bininda-Emonds et al., 2007]. This implies that the mechanism that regulates the addition of nonneuronal cells to brain structures is highly constrained, resulting in very low levels of variation in average glial cell size that are however tightly coupled to larger variations in average neuronal cell size across mammalian brain structures and species. We have proposed that this condition is enough to lead to a universal relationship between brain structure mass and numbers of glial cells, as well as between glia/ neuron ratios and average neuronal cell size, through the selfregulated addition of numbers of glial cells to the developing tissue [Mota and Herculano-Houzel, 2014].

We find that Australasian and South American marsupials share the relationship between structure mass and number of neurons that applies to each brain structure, which suggests that neuronal scaling rules within each brain structure have remained conserved in marsupial brain evolution. Moreover, we find that both marsupial groups share with afrotherians, eulipotyphlans, glires, and artiodactyls the neuronal scaling rules that apply to the $\mathrm{Cx}$, which we have previously proposed to be the ancestral neuronal scaling rule that applied at the origin of mammals [Herculano-Houzel et al., 2014a]. The conformity of marsupials to the relationship between cortical mass (including the white matter) and the number of cortical neurons that applies to eutherians is all the more remarkable given that the marsupial Cx lacks a corpus callosum, which could be expected to decrease the mass of $\mathrm{Cx}$ (including white matter) associated with a certain number of cortical neurons. However, interhemispheric connections found in the callosum of eutherians are not lacking altogether in marsupials, but rather they are bundled in the anterior commissure and fasciculus aberrans [Putnam et al., 1968; Ebner, 1969; Heath and Jones, 1971]. The neuronal scaling rules for the cerebral cortical mass thus appear constrained for a number of mammalian species, with the only exception so far being primates [Herculano-Houzel et al., 2014a]. We also find that the Cx of marsupials, at $45-64 \%$ of brain mass, is similar in relative mass to that of other mammals, in contrast to a relative mass of only $16 \%$ reported by Seelke et al. [2014] for $M$. domestica. Most importantly, we find that the Cx of marsupials contains a small percentage of about $15 \%$ of all brain neurons, in the same range as the $15-25 \%$ of all brain neurons found in the $\mathrm{Cx}$ of eutherians, regardless of the increasingly larger $\mathrm{Cx}$ within larger brains [Herculano-Houzel, 2010]. The fairly stable percentage of brain
Dos Santos et al. 
neurons in the $\mathrm{Cx}$ is explained by the coordinated, linear addition of neurons to the $\mathrm{Cx}$ and $\mathrm{Cb}$ across marsupial species as in eutherians [Herculano-Houzel, 2010].

In contrast, we find that marsupials possess their own relationships between structure mass and number of neurons for the $\mathrm{Cb}$ and $\mathrm{RoB}$. The nonconformity suggests that extant marsupials diverged from the ancestral therian scaling rules with changes that resulted in larger neuronal densities (that is, smaller neurons) in the $\mathrm{Cb}$ and smaller neuronal densities (that is, larger neurons) in the RoB compared to eutherian species with similar numbers of neurons in these structures.

Importantly, whereas neuronal scaling rules within brain structures are shared across South America and Australasian marsupials, neuronal scaling rules across structures appear to differ across the two groups. South American marsupials share with later derived afrotherians, eulipotyphlans, and glires the scaling of numbers of neurons in the $\mathrm{Cx}$ and $\mathrm{Cb}$ over the $\mathrm{RoB}$, in line with our previous suggestion that this shared relationship represents the ancestral condition for therians. In contrast, Australasian marsupials seem to have diverged from those relationships, with higher $\mathrm{N}_{C X} / \mathrm{N}_{\mathrm{ROB}}$ and $\mathrm{N}_{\mathrm{CB}} / \mathrm{N}_{\mathrm{ROB}}$ ratios like those found in primates and artiodactyls that cannot simply be predicted from their larger brain size. That is, Australasian marsupials appear to have become similar to primates and artiodactyls in that they have larger numbers of neurons allocated to the $\mathrm{Cx}$ and $\mathrm{Cb}$ over the $\mathrm{RoB}$, while the two former structures continue to gain neurons in a coordinated fashion. Verifying this possibility will require examining American marsupial species with brains as large as those of the Australasian species analyzed here. Still, the finding that the $\mathrm{Cx}$ of Australasian marsupials shares its scaling of mass as a function of number of neurons with nonprimate eutherians (including artiodactyls) while it gains neurons faster than the RoB compared to South American marsupials and to the same nonprimate eutherian species (and as fast as in primates) points to a dissociation between the developmental mechanisms that tie numbers of neurons to neuronal cell size and therefore neuronal density (and thus determine the final mass of a brain structure such as $\mathrm{Cx}$ ) and those mechanisms that regulate the allocation of neurons to different brain structures (for instance, by regulating the size of the initial progenitor pool of each structure). This is in agreement with our recent suggestion that mammalian brain evolution has occurred through both concerted and mosaic changes in those cellular mechanisms that link neuronal proliferation to average cell size within and across structures [Herculano-Houzel et al., 2014a].

Marsupial Brains Are Not Primitive
One of the ways in which marsupials diverge from eutherians is in their strikingly low neuronal densities in the RoB for the numbers of neurons in the structure. We showed that these low neuronal densities in the RoB are, however, expected for the body mass of these species, in agreement with our previous suggestion that the average mass of neurons in the RoB scales with body length (which requires longer axons and thus larger neurons in brainstem structures [Herculano-Houzel et al., 2015a]). The apparent incongruity in RoB neuronal density can thus be explained if marsupials diverged from other therians in that they have much fewer neurons in the RoB than expected for the body mass of a therian. Importantly, these findings strengthen our proposition that there is not a single relationship between numbers of brain neurons and body mass, which implies that larger bodies do not necessarily require larger numbers of neurons in a particular scaling relationship to operate them [HerculanoHouzel et al., 2015a].

\section{What Marsupials Tell Us about Ancestral \\ Mammalian Brain and Brain Evolution}

Here we show that, despite an early emergence in mammalian evolution, marsupials are derived compared to eutherians in the relationship between $\mathrm{N}_{\mathrm{ROB}}$ and $\mathrm{M}_{\mathrm{BD}}$, and in the neuronal scaling rules that apply to the $\mathrm{Cb}$ and $\mathrm{RoB}$, and Australasian marsupials are further derived in the allocation of neurons to the $\mathrm{Cx}$ and $\mathrm{Cb}$ over the RoB. Given these divergences, our findings imply that marsupials as a whole are not as "ancestral-like" as would be expected from their early divergence from eutherians in mammalian evolutionary history, and they cannot be considered extant proxies of ancestral mammalian species as previously suggested by Pirlot [1986]. While it is commonly argued that the $\mathrm{Cx}$ of American marsupial species may reflect the size, gross anatomy, and connectivity of the early mammalian cortex [Kemp, 2004; Kaas, 2011a, b], Ashwell [2008] showed that encephalization levels of Australasian species are comparable to those of eutherian mammals and even those of prosimian primates. We thus join Pirlot [1986] in arguing that extant marsupials as a whole should no longer be considered universally as proxies for ancestral mammalian brains. While we find that South American and Australasian marsupials have a neuronal composition of the $\mathrm{Cx}$ that is indeed shared with all extant nonprimate species, and are thus likely to reflect the composition of the ancestral mammalian $\mathrm{Cx}$, all other characteristics examined show enough derivation to make extant marsupials an inappropriate proxy for the ancestral mammalian brain as a whole. 


\section{Acknowledgments}

We thank Kamila Avelino De Souza, Débora Messeder Jardim, Lissa Ventura Antunes, and Kleber Neves for help in obtaining cell counts, Nina Patzke and Felix Ströckens for help with perfusions and dissections of the South American specimens of this study, Mads Bertelsen for supplying the Tasmanian devil specimen, Laura Trice for the wallaby brain sections, and Jon Kaas for making space available in his laboratory to do the immunohistochemistry for NeuN. This work was supported by the Brazilian National Council for Scientific and Technological Development (CNPq), the Foundation for Research Support of the State of Rio de Janeiro (FAPERJ), and the James S. McDonnell Foundation (S.H.-H., grant No. 220020232; C.C.S., grant No. 220020293). The funders had no role in the study design, data collection and analysis, the decision to publish, or the preparation of this paper.

\section{Disclosure Statement}

The authors declare no conflict of interests.

\section{References}

Ashwell KWS (2008): Encephalization of Australian and New Guinean marsupials. Brain Behav Evol 71:181-199.

-Ashwell KWS, McAllan BM, Mai JK, Paxinos G (2008): Cortical cyto- and chemoarchitecture in three small Australian marsupial carnivores: Sminthopsis macroura, Antechinus stuartii and Phascogale calura. Brain Behav Evol 72:215-232.

-Azevedo FAC, Carvalho LRB, Grinberg LT, Farfel JM, Ferretti REL, Leite REP, Jacob Filho W, Lent R, Herculano-Houzel S (2009): Equal numbers of neuronal and nonneuronal cells make the human brain an isometrically scaled-up primate brain. J Comp Neurol 513: 532-541.

Bininda-Emonds ORP, Cardillo M, Jones KE, MacPhee RDE, Beck RMD, Grenyer R, Price SA, Vos RA, Gittleman JL, Purvis A (2007): The delayed rise of present-day mammals. Nature 29;446:507-512.

Ebner FF (1969): A comparison of primitive forebrain organization in Metatherian and Eutherian mammals. Ann NY Acad Sci 167:241257.

- Gabi M, Collins CE, Wong P, Torres LB, Kaas JH, Herculano-Houzel S (2010): Cellular scaling rules for the brains of an extended number of primate species. Brain Behav Evol 76:32-44

- Gallus S, Janke A, Kumar V, Nilsson MA (2015): Disentangling the relationship of the Australian marsupial orders using retrotransposon and evolutionary network analyses. Genome Biol Evol 7:985-992.

Gittins R, Harrison PJ (2004): Neuronal density, size and shape in the human anterior cingulate cortex: a comparison of Nissl and NeuN staining. Brain Res Bull 63:155-160.

Haug H (1987): Brain sizes, surfaces, and neuronal sizes of the cortex cerebri: a stereological investigation of man and his variability and a comparison with some mammals (primates, whales, marsupials, insectivores, and one elephant). Am J Anat 180:126-142.

- Heath CJ, Jones EG (1971): Interhemispheric pathways in the absence of a corpus callosum: an experimental study of commissural connections in the marsupial phalanger. J Anat 109:253-270.
Herculano-Houzel S (2010): Coordinated scaling of cortical and cerebellar numbers of neurons. Front Neuroanat 4:12.

Herculano-Houzel S (2014): The glia/neuron ratio: how it varies uniformly across brain structures and species and what that means for brain physiology and evolution. Glia 62: 1377-1391.

Herculano-Houzel S, Avelino-de-Souza K, Neves K, Porfirio J, Messeder D, Mattos Feijo L, Maldonado J, Manger PR (2014b): The elephant brain in numbers. Front Neuroanat 8 : 46.

Herculano-Houzel S, Bartheld von CS, Miller DJ, Kaas JH (2015b): How to count cells: the advantages and disadvantages of the isotropic fractionator compared with stereology. Cell Tissue Res 360:29-42.

-Herculano-Houzel S, Catania K, Manger PR, Kaas JH (2015a): Mammalian brains are made of these: a dataset of the numbers and densities of neuronal and nonneuronal cells in the brain of glires, primates, scandentia, eulipotyphlans, afrotherians and artiodactyls, and their relationship with body mass. Brain Behav Evol 86:145-163.

Herculano-Houzel S, Collins CE, Wong P, Kaas JH (2007): Cellular scaling rules for primate brains. Proc Natl Acad Sci USA 104:35623567.

Herculano-Houzel S, Lent R (2005): Isotropic fractionator: a simple, rapid method for the quantification of total cell and neuron numbers in the brain. J Neurosci 25:2518-2521.

-Herculano-Houzel S, Manger PR, Kaas JH (2014a): Brain scaling in mammalian evolution as a consequence of concerted and mosaic changes in numbers of neurons and average neuronal cell size. Front Neuroanat 8:77.

Herculano-Houzel S, Messeder DJ, Fonseca-Azevedo K, Pantoja NA (2015c) When larger brains do not have more neurons: increased numbers of cells are compensated by decreased average cell size across mouse individuals. Front Neuroanat 9:64.

Herculano-Houzel S, Mota B, Lent R (2006): Cellular scaling rules for rodent brains. Proc Natl Acad Sci USA 103:12138-12143.
Herculano-Houzel S, Ribeiro P, Campos L, Valotta da Silva A, Torres LB, Catania KC, Kaas JH (2011): Updated neuronal scaling rules for the brains of glires (rodents/lagomorphs). Brain Behav Evol 78:302-314.

Kaas JH (2011a): Reconstructing the areal organization of the neocortex of the first mammals. Brain Behav Evol 78:7-21.

Kaas JH (2011b): Neocortex in early mammals and its subsequent variations. Ann NY Acad Sci 1225:28-36.

Kazu RS, Maldonado J, Mota B, Manger PR, Herculano-Houzel S (2014): Cellular scaling rules for the brain of Artiodactyla include a highly folded cortex with few neurons. Front Neuroanat $8: 128$.

Kemp TS (2004): The Origin and Evolution of Mammals. Oxford, Oxford University Press.

- Manger PR, Pillay P, Maseko BC, Bhagwandin A, Gravett N, Moon D-J, Jillani N, Hemingway J (2009): Acquisition of brains from the African elephant (Loxodonta africana): perfusion-fixation and dissection. J Neurosci Methods 179: $16-21$.

May-Collado LJ, Kilpatrick CW, Agnarsson I (2015): Mammals from "down under": a multi-gene species-level phylogeny of marsupial mammals (Mammalia, Metatheria). PeerJ 3:e805.

Mota B, Herculano-Houzel S (2014): All brains are made of this: a fundamental building block of brain matter with matching neuronal and glial masses. Front Neuroanat 8:1-21.

Mullen RJ, Buck CR, Smith AM (1992): NeuN, a neuronal specific nuclear protein in vertebrates. Development 116:201-211.

Murphy WJ, Eizirik E, Johnson WE, Zhang YP (2001): Molecular phylogenetics and the origins of placental mammals. Nature 409:614618.

Murphy WJ, Pevzner PA, O'Brien SJ (2004): Mammalian phylogenomics comes of age. Trends Genet 20:631-639.

Neves K, Ferreira FM, Tovar-Moll F, Gravett N, Bennett NC, Kaswera C, Gilissen E, Manger PR, Herculano-Houzel S (2014): Cellular scaling rules for the brain of afrotherians. Front Neuroanat 8:5. 
Ngwenya A, Patzke N, Manger PR, HerculanoHouzel S (2016): Continued growth of the central nervous system without mandatory addition of neurons in the Nile crocodile (Crocodylus niloticus). Brain Behav Evol 87: 19-38.

Nilsson MA, Churakov G, Sommer M, Tran NV, Zemann A, Brosius J, Schmitz J (2010): Tracking marsupial evolution using archaic genomic retroposon insertions. PLoS Biol 8:e1000436.

-Olkowicz S, Kocourek M, Lucan R, Portes M, Herculano-Houzel S, Nemec P (2016): Birds have primate-like numbers of neurons in the telencephalon. Proc Natl Acad Sci USA 113:7255-7260

- Pirlot P (1986): Understanding taxa by comparing brains. Perspect Biol Med 29:499-509.

- Putnam SJ, Megirian D, Manning JW (1968): Marsupial interhemispheric relation. J Comp Neurol 132:227-234.
Rosa M, Krubitzer LA, Molnar Z, Nelson JE (1999): Organization of visual cortex in the northern quoll, Dasyurus hallucatus: evidence for a homologue of the second visual area in marsupials. Eur J Neurosci 11:907915.

arko DK, Catania KC, Leitch DB, Kaas JH, Herculano-Houzel S (2009): Cellular scaling rules of insectivore brains. Front Neuroanat 3:8.

Saunders NR, Adam E, Reader M, Møllgård K (1989): Monodelphis domestica (grey shorttailed opossum): an accessible model for studies of early neocortical development. Anat Embryol 180:227-236.

Seelke AMH, Dooley JC, Krubitzer LA (2013): Differential changes in the cellular composition of the developing marsupial brain. J Comp Neurol 521:2602-2620.
-Seelke AMH, Dooley JC, Krubitzer LA (2014): The cellular composition of the marsupial neocortex. J Comp Neurol 522:2286-2298.

Sikes RS, Gannon WL (2011): Guidelines of the American Society of Mammalogists for the use of wild mammals in research. J Mammal 92:235-253.

Stolzenburg J-U, Reichenbach A, Neumann M (1989): Size and density of glial and neuronal cells within the cerebral neocortex of various insectivorian species. Glia 2:78-84.

Watson C, Provis J, Herculano-Houzel S (2012): What determines motor neuron number? Slow scaling of facial motor neuron numbers with body mass in marsupials and primates. Anat Rec 295:1683-1691.

Wong P, Kaas JH (2009): An architectonic study of the neocortex of the short-tailed opossum (Monodelphis domestica). Brain Behav Evol 73:206-228. 


\section{Erratum}

In the article by Dos Santos SE et al., entitled "Cellular Scaling Rules for the Brain of Marsupials: Not as 'Primitive' as Expected” [Brain Behav Evol 2017;89:48-63, DOI: 10.1159/ 000452856], the following values have to be corrected:

The correct number of neurons in the cerebral cortex $\left(\mathrm{N}_{\mathrm{CX}}\right)$ of the Tasmanian devil (Sarcophilus) in online supplementary Table S1 is $71.66 \times 10^{6}$ (for online suppl. material, see www.karger.com/doi/10.1159/000452856).

The conclusions of the paper are in no way impacted. The correction only impacts the values listed below (as well as Figure $5 \mathrm{a}-\mathrm{c}$, see below):

- The cerebral cortex holds $15.2 \pm 1.2 \%$ of all brain neurons.

- Larger marsupial brains do not have a greater percentage of their neurons located in the cerebral cortex (Spearman, $p=0.5755$ ).

- Larger cortices do not have proportionally more neurons (Spearman, $p=0.3807$ ).

- The hippocampus holds on average $11.5 \pm 2.0 \%$ of all cortical neurons.

- The correlation between cortical mass and the percentage of cortical neurons located in the hippocampus does not reach significance $(p=0.1544)$.

- The relationship between $\mathrm{N}_{\mathrm{CB}}$ and $\mathrm{N}_{\mathrm{CX}}$ is a significantly linear function with $r^{2}=0.926$ $(p<0.0001)$ or a power function of exponent $0.917 \pm 0.091(p<0.0001)$.

- The average $\mathrm{N}_{C X} / \mathrm{N}_{\mathrm{ROB}}$ ratio in Australasian marsupials is $5.8 \pm 0.7$ and is not significantly different from the ratio found in artiodactyls (Wilcoxon, $p=0.6481$ ). 


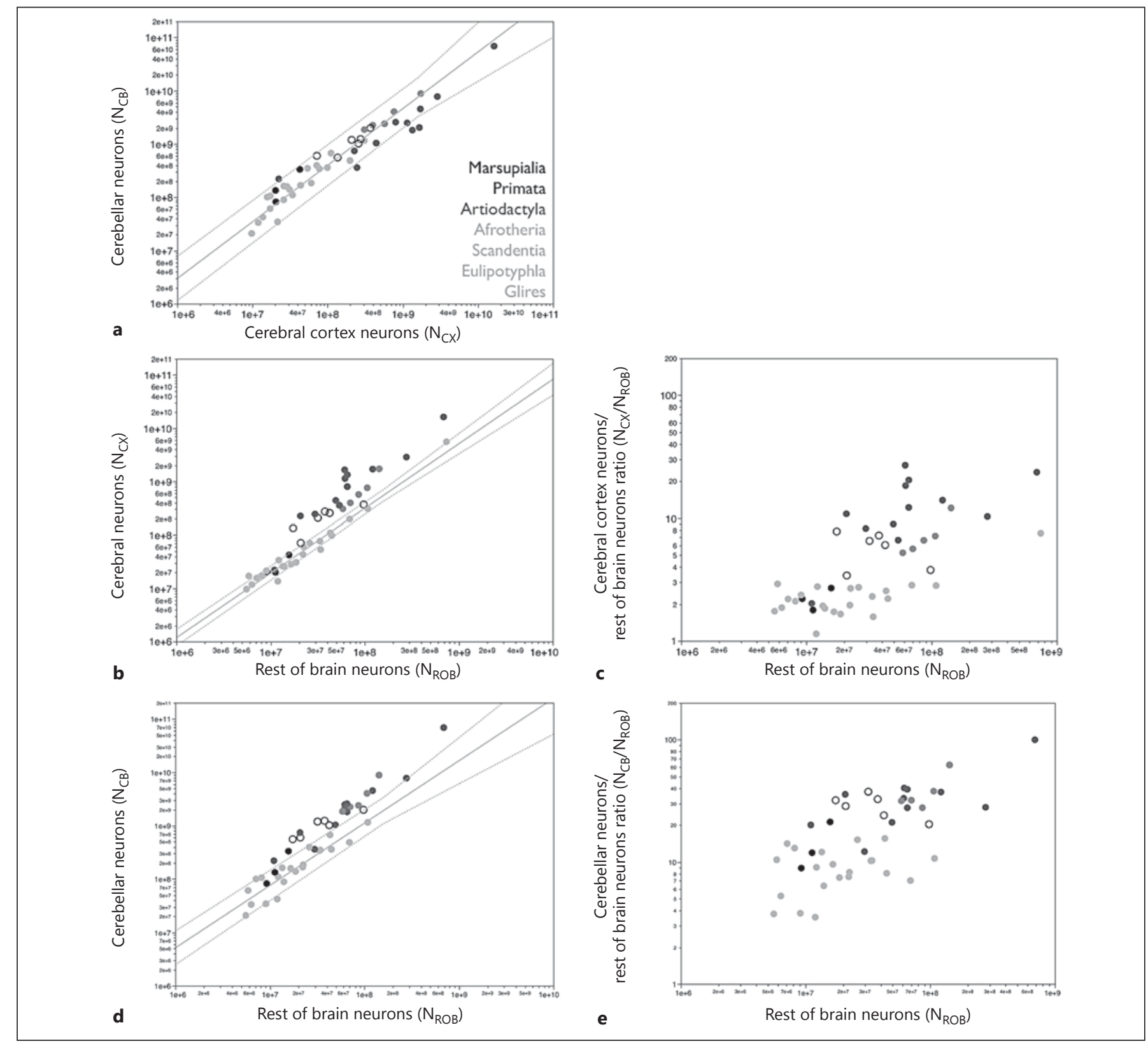

Fig. 5. Relative increase in numbers of neurons in the cerebral cortex and cerebellum of Australasian marsupials, with a shared scaling of numbers of neurons across these structures. a Scaling of $\mathrm{N}_{\mathrm{CB}}$ as a function of $\mathrm{N}_{\mathrm{CX}}$ varies in a similar way for all theria with exponents near linearity: $0.917 \pm 0.091$ in marsupials, $0.867 \pm 0.108$ in primates, $0.923 \pm 0.110$ in artiodactyls, and $1.063 \pm 0.111$ in afrotherians, glires, and scandentian together (plotted line). b The $\mathrm{N}_{\mathrm{CX}}$ in American marsupials matches the expected for $\mathrm{N}_{\mathrm{ROB}}$ (closed black symbols), while Australasian marsupial species have much higher $\mathrm{N}_{C X}$ than expected for their $\mathrm{N}_{\mathrm{ROB}}$ (open symbols). c Accordingly, $\mathrm{N}_{\mathrm{CX}} / \mathrm{N}_{\mathrm{ROB}}$ is higher in Australasian marsupials $(5.8 \pm 0.7)$ than in South American marsupials $(2.2 \pm 0.3)$, making the latter comparable to all nonprimate, nonartiodactyla theria and the former comparable to Artiodactyla $\left(\mathrm{N}_{\mathrm{CX}} / \mathrm{N}_{\mathrm{ROB}}=7.3 \pm 1.2\right)$. d $\mathrm{N}_{\mathrm{CB}}$ varies as a power function of $\mathrm{N}_{\mathrm{ROB}}$ of exponent $1.334 \pm 0.212$ in marsupials, $1.315 \pm 0.112$ in Primata, $1.737 \pm 0.305$ in Artiodactyla, and $1.169 \pm 0.116$ in Afrotheria (minus the elephant), Glires, Eulipotyphla and Scandentia together (plotted line). e $\mathrm{N}_{\mathrm{CB}} / \mathrm{N}_{\mathrm{ROB}}$ is higher in Australasian marsupials $(29.2 \pm 2.5)$ than in South American marsupials $(14.0 \pm 3.7)$, making the former comparable to Artiodactyla and Primata $\left(\mathrm{N}_{\mathrm{CB}} / \mathrm{N}_{\mathrm{ROB}}=38.3 \pm 6.2\right.$ and $35.9 \pm 7.0$, respectively) and the latter significantly different from its Australasian counterpart but also from Afrotheria, Glires, Eulipotyphla and Scandentia together ( $p=0.0112$ and $p=0.0423$, respectively). Species are shown in shades of gray as displayed in a. Australasian marsupials (open symbols) and South American marsupials (closed symbols). Values are exponents \pm SE. Data are from Herculano-Houzel et al. [2006, 2007, 2011, 2014b], Azevedo et al. [2009], Sarko et al. [2009], Gabi et al. [2010], Kazu et al. [2014], and Neves et al. [2014]. 